\title{
HERMITIAN-EINSTEIN CONNECTIONS ON PRINCIPAL BUNDLES OVER FLAT AFFINE MANIFOLDS
}

\author{
INDRANIL BISWAS AND JOHN LOFTIN
}

\begin{abstract}
Let $M$ be a compact connected special flat affine manifold without boundary equipped with a Gauduchon metric $g$ and a covariant constant volume form. Let $G$ be either a connected reductive complex linear algebraic group or the real locus of a split real form of a complex reductive group. We prove that a flat principal $G$-bundle $E_{G}$ over $M$ admits a Hermitian-Einstein structure if and only if $E_{G}$ is polystable. A polystable flat principal $G$-bundle over $M$ admits a unique Hermitian-Einstein connection. We also prove the existence and uniqueness of a Harder-Narasimhan filtration for flat vector bundles over $M$. We prove a Bogomolov type inequality for semistable vector bundles under the assumption that the Gauduchon metric $g$ is astheno-Kähler.
\end{abstract}

\section{INTRODUCTION}

A flat affine manifold $M$ is a $C^{\infty}$ manifold equipped with a flat torsionfree connection on $T M$. Equivalently, a flat affine structure on a manifold is provided by an atlas of coordinate charts whose transition functions are all affine maps $x \mapsto A x+b$. The total space of the tangent bundle $T M$ of a flat affine manifold admits a complex structure, with the transition maps being $z \mapsto A z+b$, for $z=x+\sqrt{-1} y$, with $y$ representing the fiber coordinates. There is a dictionary between holomorphic objects on $T M$ which are invariant in the fiber directions and locally constant objects on $M$ (cf. [Lo]). This correspondence between affine and complex manifolds has recently become prominent as a part of the mirror conjecture of Strominger-Yau-Zaslow (in this case, each fiber of the tangent bundle $T M \longrightarrow M$ is quotiented by a lattice to form a special Lagrangian torus in a Calabi-Yau manifold). In particular, a flat vector bundle over $M$ naturally extends to a holomorphic vector bundle over $T M$.

We briefly recall the set-up and the main result of [Lo]. An affine manifold $M$ is called special if the induced flat connection on the line bundle $\bigwedge^{\text {top }} T M$ has trivial holonomy. Let $M$ be a compact connected special flat affine manifold without boundary. Fix a nonzero flat section $\nu$ of $\wedge^{\text {top }} T M$ (under our dictionary, $\nu$ corresponds to a holomorphic volume form on the total space of $T M$ ). Also fix an affine Gauduchon metric $g$ on $M$. This allows us to define the degree of a flat vector bundle over $M$ (see Section 2 below); we will consider both real and complex vector bundles. By a flat vector bundle we will always mean a vector bundle equipped with a flat connection (and thus locally constant

2000 Mathematics Subject Classification. 53C07.

Key words and phrases. Flat affine manifold, Hermitian-Einstein connection, principal bundle, Harder-Narasimhan filtration. 
transition functions). For a flat vector bundle $V \longrightarrow M$, its degree is denoted by $\operatorname{deg}_{g}(V)$, and its slope $\operatorname{deg}_{g}(V) / \operatorname{rank}(V)$ is denoted by $\mu_{g}(V)$.

Once degree is defined, we can define semistable, stable and polystable flat vector bundles over $M$ by imitating the corresponding definitions for holomorphic vector bundles over compact Gauduchon manifolds. Similarly, Hermitian-Einstein metrics on flat vector bundles over $M$ are defined by imitating the definition of Hermitian-Einstein metrics on holomorphic vector bundles over Gauduchon manifolds.

The following theorem is proved in [Lo, with the main technical part being to use estimates to prove a version of the Donaldson-Uhlenbeck-Yau Theorem on existence of Hermitian-Einstein connections on stable vector bundles.

Theorem 1.1 ([Lo]). A flat vector bundle $V$ over $M$ admits a Hermitian-Einstein metric if and only if $V$ is polystable. A polystable flat vector bundle admits a unique HermitianEinstein connection.

Our aim here is to establish a similar result for flat principal bundles over $M$.

Let $G$ be a connected Lie group such that it is either a complex reductive linear algebraic group or it is the fixed point locus of an anti-holomorphic involution $\sigma_{G_{\mathbb{C}}}: G_{\mathbb{C}} \longrightarrow G_{\mathbb{C}}$, where $G_{\mathbb{C}}$ is a complex reductive linear algebraic group; if $G$ is of the second type, then we assume that $\sigma_{G_{\mathbb{C}}}$ is of split type. Extending the definition of a flat polystable vector bundle on $M$, we define polystable flat principal $G$-bundles over $M$. A flat principal $\mathrm{GL}_{r}$-bundle is polystable if and only if the corresponding vector bundle of rank $r$ is polystable.

Fix a maximal compact subgroup $K \subset G$. A Hermitian structure on a flat principal $G$-bundle $E_{G}$ on $M$ is a $C^{\infty}$ reduction of structure group $E_{K} \subset E_{G}$ to the subgroup $K$. If $G$ is the real points of $G_{\mathbb{C}}$, given a flat principal $G$-bundle $E_{G}$ over $M$, we get a holomorphic principal $G_{\mathbb{C}}$-bundle $E_{G_{\mathbb{C}}}$ over the total space of $T M$. For any Hermitian structure on $E_{G}$, there is a naturally associated connection on the principal $G_{\mathbb{C}}$-bundle $E_{G_{\mathbb{C}}}$.

A Hermitian structure on $E_{G}$ produces a connection on $E_{G}$. Contracting using $g$ the curvature form of the connection, we obtain a section of the adjoint bundle $\operatorname{ad}\left(E_{G}\right)$. A Hermitian structure on $E_{G}$ is called Hermitian-Einstein if this section of $\operatorname{ad}\left(E_{G}\right)$ is given by some element in the center of $\operatorname{Lie}(G)$. The connection associated to a Hermitian structure satisfying this condition is called a Hermitian-Einstein connection.

We prove the following generalization of Theorem 1.1 (see Theorem 6.1):

Theorem 1.2. A flat principal $G$-bundle $E_{G} \longrightarrow M$ admits a Hermitian-Einstein structure if and only if $E_{G}$ is polystable. A polystable flat principal $G$-bundle admits a unique Hermitian-Einstein connection.

We prove the following analog of the Harder-Narasimhan filtration (see Theorem 2.5):

Theorem 1.3. For any flat vector bundle $V \longrightarrow M$, there is a unique filtration of flat subbundles

$$
0=F_{0} \subset F_{1} \subset \cdots \subset F_{\ell-1} \subset F_{\ell}=V
$$


such that for each $i \in[1, \ell]$, the flat vector bundle $F_{i} / F_{i-1}$ is semistable, and

$$
\mu_{g}\left(F_{1}\right)>\mu_{g}\left(F_{2} / F_{1}\right)>\cdots>\mu_{g}\left(F_{\ell} / F_{\ell-1}\right) .
$$

Our proof of Theorem 1.2 crucially uses Theorem 1.3 .

One goal of the present work and of [Lo] is to develop analytic tools to study affine manifolds. In the complex case, Li-Yau-Zheng and also Teleman have used HermitianEinstein metrics on vector bundles over non-Kähler surfaces equipped with Gauduchon metrics to partially classify surfaces of Kodaira class VII [LYZ, Te94, Te05]. There are similar open problems in classifying affine manifolds in low dimension. As suggested by Bill Goldman, one case that may be tractable is that of flat affine symplectic fourmanifolds (flat affine four-manifolds admitting a flat nondegenerate closed two-form). Little is known about these manifolds.

In particular, we hope to use the results of this paper to study representations of $\pi_{1}(M)$ into a reductive Lie group $G$. It is well known that a flat principal $G$-bundle over a manifold $M$ is equivalent to a conjugacy class of homomorphisms from $\pi_{1}(M)$ to $G$. Our Theorem 1.2 thus can be rephrased as follows: If $M$ is a special affine manifold equipped with a Gauduchon metric $g$, every representation $\pi_{1}(M) \longrightarrow G$ admits either a nontrivial destabilizing subrepresentation or a unique Hermitian-Einstein connection.

For a general affine manifold $M$ and representation $\pi_{1}(M) \longrightarrow G$, we expect there to be few subrepresentations at all, and so the existence of the canonical Hermitian-Einstein connection is to be expected in many cases.

In Section 6.2, we prove the following Bogomolov type inequality as an application of Theorem 1.2 (see Lemma 6.2):

Lemma 1.4. Assume that the Gauduchon metric $g$ satisfies the condition that $\partial \bar{\partial}\left(\omega_{g}^{d-2}\right)=$ 0 , where $\omega$ is the corresponding $(1,1)$-form (it is called an astheno-Kähler metric). Let $V \longrightarrow M$ be a semistable flat vector bundle of rank $r$. Then

$$
\int_{M} \frac{c_{2}(\mathcal{E} n d(V)) \omega_{g}^{d-2}}{\nu}=\int_{M} \frac{\left(2 r \cdot c_{2}(V)-(r-1) c_{1}(V)^{2}\right) \omega_{g}^{d-2}}{\nu} \geq 0 .
$$

A few notes about the proof are in order. As in [Lo], we are able to follow the existing proofs in the complex case closely, with a few important simplifications. In [Lo], the main simplification is that we need only consider flat subbundles (as opposed to singular subsheaves) as destabilizing objects in non-stable vector bundles. In the current work, we find another such simplification in the proof of Lemma 2.2. Flatness implies that the space of all flat subbundles of rank $k$ of a given flat vector bundle $V$ is a closed subset of the Grassmannian of the fiber of $V$ at a given point in $M$, and thus a simple compactness argument guarantees the existence of a flat subbundle with maximal slope. The corresponding argument in the complex Gauduchon case is more complicated $[\mathrm{Br}]$.

\section{HARDER-NARASimhan Filtration of A VECTOR BUNDLE}

We recall from [Lo] some basic definitions. Consider a flat affine manifold $M$ of dimension $n$ as the zero section of its tangent bundle $T M$. The unifying idea behind all 
these definitions is to consider flat objects on $M$ to be restrictions of holomorphic objects on $T M$. We may define operators $\partial$ and $\bar{\partial}$ on the affine Dolbeault complex, where $(p, q)$ forms are represented as sections of $\Lambda^{p}\left(T^{*} M\right) \otimes \Lambda^{q}\left(T^{*} M\right)$. A Riemannian metric $g$ on $M$ can be extended to a natural Hermitian metric on the total space of $T M$. Let $\omega_{g}$ be the associated $(1,1)$ form. The metric $g$ is called affine Gauduchon if $\partial \bar{\partial}\left(\omega_{g}^{n-1}\right)=0$. Given a flat vector bundle $V \longrightarrow M$ equipped with a Hermitian bundle metric $h$, we may define its first Chern form $c_{1}(h)$. Assume there is a covariant constant volume form $\nu$ on $M$. Define the degree of the vector bundle as

$$
\operatorname{deg}_{g}(V):=\int_{M} \frac{c_{1}(h) \wedge \omega_{g}^{n-1}}{\nu} .
$$

Then the slope of $V$ is defined to be

$$
\mu_{g}(V):=\operatorname{deg}_{g}(V) / \operatorname{rank}(V) .
$$

The vector bundle $V$ is said to be stable if every flat subbundle $W$ of $V$ with $0<$ $\operatorname{rank}(W)<\operatorname{rank}(V)$ satisfies the inequality $\mu_{g}(W)<\mu_{g}(V)$. The vector bundle $V$ is called semistable if $\mu_{g}(W) \leq \mu_{g}(V)$ for all such $W$, and $V$ is said to be polystable if it is a direct sum of stable flat bundles of the same slope.

Let

$$
V \longrightarrow M
$$

be a flat vector bundle; it is allowed to be real or complex. Fix a filtration of flat subbundles

$$
0=V_{0} \subset V_{1} \subset \cdots \subset V_{n-1} \subset V_{n}=V
$$

such that all the successive quotients $V_{i} / V_{i-1}, 1 \leq i \leq n$, are stable.

Define

$$
\delta:=\operatorname{Max}\left\{\mu_{g}\left(V_{i} / V_{i-1}\right)\right\}_{i=1}^{n} \in \mathbb{R} .
$$

Lemma 2.1. Let $F \subset V$ be any flat subbundle of $V$ of positive rank. Then $\mu_{g}(F) \leq \delta$.

Proof. If $F$ is not semistable, then there is a flat subbundle $F^{\prime} \subset F$ such that

$$
0<\operatorname{rank}\left(F^{\prime}\right)<\operatorname{rank}(F) \text { and } \mu_{g}\left(F^{\prime}\right)>\mu_{g}(F) \text {. }
$$

Furthermore, if $F$ is semistable, then there is a flat subbundle $F^{\prime \prime} \subset F$ such that $\operatorname{rank}\left(F^{\prime \prime}\right)$ is smallest among the ranks of all flat subbundles $W$ of $F$ with $\mu_{g}(W)=\mu_{g}(F)$. Note that such a smallest rank flat vector bundle $F^{\prime \prime}$ is automatically stable. Therefore, it is enough to check the inequality in the lemma under the assumption that $F$ is stable.

Assume that $F$ is stable, and $\mu_{g}(F)>\delta$.

Let $F_{1}$ and $F_{2}$ be semistable flat vector bundles over $M$ such that either both of them are real or both are complex. Let

$$
\varphi: F_{1} \longrightarrow F_{2}
$$

be a nonzero flat homomorphism of vector bundles. Then

$$
\mu_{g}\left(F_{1}\right) \leq \mu_{g}(\operatorname{Image}(\varphi)) \leq \mu_{g}\left(F_{2}\right)
$$


because $F_{1}$ and $F_{2}$ are semistable. Therefore, there is no nonzero flat homomorphism of vector bundles from $F_{1}$ to $F_{2}$ if $\mu_{g}\left(F_{1}\right)>\mu_{g}\left(F_{2}\right)$.

From the above observation we conclude that for each $i \in[1, n]$, there is no nonzero flat homomorphism of vector bundles from $F$ to $V_{i} / V_{i-1}$. This immediately implies that there is no nonzero flat homomorphism of vector bundles from $F$ to $V$. This contradicts the fact that $F$ is a flat subbundle of $V$. Therefore, we conclude that $\mu_{g}(F) \leq \delta$.

Define

$$
\delta_{0}(V):=\operatorname{Sup}\left\{\mu_{g}(F) \mid F \text { is a flat subbundle of } V\right\}
$$

which is a finite number due to Lemma 2.1.

Lemma 2.2. There is a flat subbundle $F \subset V$ such that $\mu_{g}(F)=\delta_{0}(V)$, where $\delta_{0}(V)$ is defined in (2.3).

Proof. Fix an integer $k \in[1, \operatorname{rank}(V)]$ such that

$$
\delta_{0}(V)=\operatorname{Sup}\left\{\mu_{g}(F) \mid F \subset V \text { is a flat subbundle of rank } k\right\} ;
$$

such a $k$ clearly exists because $\operatorname{rank}(V)$ is a finite integer. Fix a point

$$
x_{0} \in M \text {. }
$$

Let $\operatorname{Gr}\left(V_{x_{0}}, k\right)$ be the Grassmannian parametrizing all linear subspaces of dimension $k$ of the fiber $V_{x_{0}}$.

For any flat subbundle $F \subset V$ of rank $k$, consider the subspace $F_{x_{0}} \in \operatorname{Gr}\left(V_{x_{0}}, k\right)$. We note that the flat subbundle $F$ is uniquely determined by the point $F_{x_{0}} \in \operatorname{Gr}\left(V_{x_{0}}, k\right)$, because $M$ is connected. Let

$$
S \subset \operatorname{Gr}\left(V_{x_{0}}, k\right)
$$

be the locus of all subspaces that are fibers of flat subbundles of $V$ of rank $k$. We will now describe $S$ explicitly.

Let

$$
\rho: \pi_{1}\left(M, x_{0}\right) \longrightarrow \operatorname{GL}\left(V_{x_{0}}\right)
$$

be the monodromy representation for the flat connection on $V$. The group $\operatorname{GL}\left(V_{x_{0}}\right)$ acts on $\operatorname{Gr}\left(V_{x_{0}}, k\right)$ in a natural way. The subset $S$ in (2.4) is the fixed point locus

$$
S=\operatorname{Gr}\left(V_{x_{0}}, k\right)^{\rho\left(\pi_{1}\left(M, x_{0}\right)\right)}
$$

for the action of $\rho\left(\pi_{1}\left(M, x_{0}\right)\right)$ on $\operatorname{Gr}\left(V_{x_{0}}, k\right)$. Note that

$$
S=\operatorname{Gr}\left(V_{x_{0}}, k\right)^{\overline{\rho\left(\pi_{1}\left(M, x_{0}\right)\right)}} .
$$

This implies that $S$ is a closed subset of $\operatorname{Gr}\left(V_{x_{0}}, k\right)$. In particular, $S$ is compact.

For each point $z \in S$, let $F^{z} \subset V$ be the unique flat subbundle of $V$ such that $\left(F^{z}\right)_{x_{0}}=z$. We have a continuous function

$$
f_{V}^{k}: S \longrightarrow \mathbb{R}
$$


defined by $z \longmapsto \mu_{g}\left(F^{z}\right)$. Since $S$ is compact, there is a point $z_{0} \in S$ at which the function $f_{V}^{k}$ takes the maximum value $\delta_{0}(V)$. The corresponding flat subbundle $F^{z_{0}}=F$ satisfies the condition in the lemma.

Proposition 2.3. There is a unique maximal flat subbundle $F \subset V$ with $\mu_{g}(F)=\delta_{0}(V)$, where $\delta_{0}(V)$ is defined in (2.3).

Proof. Let $F_{1}$ and $F_{2}$ be two flat subbundles of $V$ such that

$$
\mu_{g}\left(F_{1}\right)=\mu_{g}\left(F_{2}\right)=\delta_{0}(V) .
$$

Note that $F_{1}$ and $F_{2}$ are automatically semistable. Let

$$
F_{1}+F_{2} \subset V
$$

be the flat subbundle generated by $F_{1}$ and $F_{2}$. We have a short exact sequence of flat vector bundles

$$
0 \longrightarrow F_{1} \cap F_{2} \longrightarrow F_{1} \oplus F_{2} \longrightarrow F_{1}+F_{2} \longrightarrow 0 \text {. }
$$

Since $F_{1}$ and $F_{2}$ are both semistable, from (2.5) it follows immediately that $F_{1} \oplus F_{2}$ is also semistable with

$$
\mu_{g}\left(F_{1} \oplus F_{2}\right)=\delta_{0}(V)
$$

We will show that $F_{1}+F_{2}$ is semistable with $\mu_{g}\left(F_{1}+F_{2}\right)=\delta_{0}(V)$.

To prove this, first note that if $F_{1} \cap F_{2}=0$, then $F_{1} \oplus F_{2}=F_{1}+F_{2}$, hence it is equivalent to the above observation. So assume that $\operatorname{rank}\left(F_{1} \cap F_{2}\right)>0$.

From (2.6),

$$
\operatorname{deg}_{g}\left(F_{1} \oplus F_{2}\right)=\operatorname{deg}_{g}\left(F_{1} \cap F_{2}\right)+\operatorname{deg}_{g}\left(F_{1}+F_{2}\right)
$$

Hence

$$
\mu_{g}\left(F_{1} \oplus F_{2}\right)=\frac{\mu_{g}\left(F_{1} \cap F_{2}\right) \cdot \operatorname{rank}\left(F_{1} \cap F_{2}\right)+\mu_{g}\left(F_{1}+F_{2}\right) \cdot \operatorname{rank}\left(F_{1}+F_{2}\right)}{\operatorname{rank}\left(F_{1} \cap F_{2}\right)+\operatorname{rank}\left(F_{1}+F_{2}\right)} .
$$

Since $F_{1} \cap F_{2}$ and $F_{1}+F_{2}$ are flat subbundles of $V$, we have

$$
\mu_{g}\left(F_{1} \cap F_{2}\right), \mu_{g}\left(F_{1}+F_{2}\right) \leq \delta_{0}(V) .
$$

Using (2.7) and (2.8) and (2.9) we conclude that

$$
\mu_{g}\left(F_{1}+F_{2}\right)=\mu_{g}\left(F_{1} \cap F_{2}\right)=\delta_{0}(V) .
$$

Therefore, we have proved that $F_{1}+F_{2}$ is semistable with $\mu_{g}\left(F_{1}+F_{2}\right)=\delta_{0}(V)$.

Consider the flat subbundle $F \subset V$ generated by all flat subbundles $W$ with $\mu_{g}(W)=$ $\delta_{0}(V)$. Since $F_{1}+F_{2}$ is semistable with $\mu_{g}\left(F_{1}+F_{2}\right)=\delta_{0}(V)$ whenever $F_{1}$ and $F_{2}$ are semistable with slope $\delta_{0}(V)$, if follows immediately that the flat subbundle $F$ satisfies the condition in the proposition.

The unique maximal flat semistable subbundle $F \subset V$ with $\mu_{g}(F)=\delta_{0}(V)$ in Proposition 2.3 will be called the maximal semistable subbundle of $V$.

Proposition 2.3 has the following corollary: 
Corollary 2.4. There is a unique filtration of flat subbundles

$$
0=F_{0} \subset F_{1} \subset \cdots \subset F_{\ell-1} \subset F_{\ell}=V
$$

such that for each $i \in[1, \ell]$, the flat subbundle $F_{i} / F_{i-1} \subset V / F_{i-1}$ is the unique maximal semistable subbundle.

The filtration in Corollary 2.4 can be reformulated as follows:

Theorem 2.5. Let $V$ be a flat vector bundle over $M$. Then there is a unique filtration of $V$ by flat subbundles

$$
0=F_{0} \subsetneq F_{1} \subsetneq \cdots \subsetneq F_{j} \subsetneq F_{j+1} \subsetneq \cdots \subsetneq F_{\ell-1} \subsetneq F_{\ell}=V
$$

such that for each $i \in[1, \ell]$, the flat vector bundle $F_{i} / F_{i-1}$ is semistable, and

$$
\mu_{g}\left(F_{1}\right)>\mu_{g}\left(F_{2} / F_{1}\right)>\cdots>\mu_{g}\left(F_{j+1} / F_{j}\right)>\cdots>\mu_{g}\left(F_{\ell} / F_{\ell-1}\right) .
$$

Proof. The filtration in Corollary 2.4 clearly has the property that for each $i \in[1, \ell]$, the flat subbundle $F_{i} / F_{i-1}$ is semistable, and

$$
\mu_{g}\left(F_{1}\right)>\mu_{g}\left(F_{2} / F_{1}\right)>\cdots>\mu_{g}\left(F_{\ell} / F_{\ell-1}\right) .
$$

Now, let

$$
0=E_{0} \subset E_{1} \subset \cdots \subset E_{n-1} \subset E_{n}=V
$$

be another filtration of flat subbundles of $V$ such that for each $i \in[1, n]$, the flat subbundle $E_{i} / E_{i-1}$ is semistable, and

$$
\mu_{g}\left(E_{1}\right)>\mu_{g}\left(E_{2} / E_{1}\right)>\cdots>\mu_{g}\left(E_{n} / E_{n-1}\right) .
$$

To show that the filtration in Corollary 2.4 coincides with the filtration in (2.10), it suffices to prove that $E_{1}=F_{1}$, because we may replace $V$ by $V / F_{i}$ and use induction on $i$.

If $n=1$, then $V$ is semistable. Hence $F_{1}=V$, and the theorem is evident.

Hence assume that $n \geq 2$.

We have

$$
\mu_{g}\left(E_{n} / E_{n-1}\right)<\mu_{g}\left(E_{1}\right) \leq \mu_{g}\left(F_{1}\right)
$$

because $F_{1}$ is the maximal semistable subbundle of $V$. Therefore, case there is no nonzero flat homomorphism from $F_{1}$ to $E_{n} / E_{n-1}$ (see the proof of Lemma 2.1). Now, by induction, there is no nonzero flat homomorphism from $F_{1}$ to $E_{i} / E_{i-1}$ for all $i \geq 2$. Hence there is no nonzero flat homomorphism from $F_{1}$ to $V / E_{1}$. Consequently, $F_{1}$ is a subbundle of $E_{1}$.

We have $\mu_{g}\left(E_{1}\right) \geq \mu_{g}\left(F_{1}\right)$ because $E_{1}$ is semistable and $F_{1}$ is a subbundle of $E_{1}$. On the other hand, we have $\mu_{g}\left(F_{1}\right) \geq \mu_{g}\left(E_{1}\right)$ because $F_{1}$ is the maximal semistable subbundle of $V$. Therefore, $\mu_{g}\left(E_{1}\right)=\mu_{g}\left(F_{1}\right)$. Again from the fact that $F_{1}$ is the maximal semistable subbundle of $V$ we conclude that the subbundle $F_{1} \subset E_{1}$ must coincide with $E_{1}$. 


\section{Semistability of TENSOR PRODUCT}

A flat vector bundle $(V, D)$ over $M$ will be called polystable if

$$
(V, D)=\left(\bigoplus_{i=1}^{m} W_{i}, \bigoplus_{i=1}^{m} D_{i}\right),
$$

where $\left(W_{i}, D_{i}\right), 1 \leq i \leq m$, are flat stable vector bundles, and

$$
\mu_{g}\left(W_{1}\right)=\cdots=\mu_{g}\left(W_{m}\right) .
$$

Let $V_{1}$ and $V_{2}$ be flat vector bundles over $M$ such that either both are real or both are complex.

Lemma 3.1. If $V_{1}$ and $V_{2}$ are stable, then the flat vector bundle $V_{1} \otimes V_{2}$ is polystable.

Proof. Assume that $V_{1}$ and $V_{2}$ are stable. Then each one them admits an affine HermitianEinstein metric (see [Lo, p. 102, Theorem 1] for the complex case and [Lo, p. 129, Corollary 33] for the real case). Let $h_{1}$ and $h_{2}$ be affine Hermitian-Einstein metrics on $V_{1}$ and $V_{2}$ respectively. The Hermitian metric on $V_{1} \otimes V_{2}$ induced by $h_{1}$ and $h_{2}$ is clearly an affine Hermitian-Einstein one. Therefore, $V_{1} \otimes V_{2}$ is polystable [Lo, p. 110, Theorem $4]$.

Corollary 3.2. If $V_{1}$ and $V_{2}$ are polystable, then the flat vector bundle $V_{1} \otimes V_{2}$ is also polystable.

Proof. This follows from Lemma 3.1 after writing the flat polystable vector bundles $V_{1}$ and $V_{2}$ as direct sums of flat stable vector bundles.

Proposition 3.3. If $V_{1}$ and $V_{2}$ are semistable, then the flat vector bundle $V_{1} \otimes V_{2}$ is semistable.

Proof. First assume that $V_{2}$ is stable. Let

$$
0=W_{0} \subset W_{1} \subset \cdots \subset W_{n-1} \subset W_{n}=V_{1}
$$

be a filtration of flat subbundles such that each successive quotient $W_{i} / W_{i-1}, 1 \leq i \leq n$, is stable with $\mu_{g}\left(W_{i} / W_{i-1}\right)=\mu_{g}\left(V_{1}\right)$. Consider the filtration of flat subbundles

$$
0=W_{0} \otimes V_{2} \subset W_{1} \otimes V_{2} \subset \cdots \subset W_{n-1} \otimes V_{2} \subset W_{n} \otimes V_{2}=V_{1} \otimes V_{2}
$$

obtained by tensoring the filtration in (3.1) by $V_{2}$. Each successive quotient in this filtration is polystable by Lemma 3.1; also,

$$
\mu_{g}\left(\left(W_{i} / W_{i-1}\right) \otimes V_{2}\right)=\mu_{g}\left(W_{i} / W_{i-1}\right)+\mu_{g}\left(V_{2}\right)=\mu_{g}\left(V_{1}\right)+\mu_{g}\left(V_{2}\right) .
$$

In view of these properties of the successive quotients for the filtration in (3.2) we conclude that $V_{1} \otimes V_{2}$ is semistable.

If $V_{2}$ is not stable, then fix a filtration

$$
0=W_{0}^{\prime} \subset W_{1}^{\prime} \subset \cdots \subset W_{m-1}^{\prime} \subset W_{m}^{\prime}=V_{2}
$$


such that each successive quotient $W_{i}^{\prime} / W_{i-1}^{\prime}, 1 \leq i \leq m$, is stable, and $\mu_{g}\left(W_{i}^{\prime} / W_{i-1}^{\prime}\right)=$ $\mu_{g}\left(V_{2}\right)$. Consider the filtration of $V_{1} \otimes V_{2}$

$$
0=V_{1} \otimes W_{0}^{\prime} \subset V_{1} \otimes W_{1}^{\prime} \subset \cdots \subset V_{1} \otimes W_{m-1}^{\prime} \subset V_{1} \otimes W_{m}^{\prime}=V_{1} \otimes V_{2}
$$

obtained by tensoring the above filtration with $V_{1}$. For each $1 \leq i \leq m$, the quotient

$$
\left(V_{1} \otimes W_{i}^{\prime}\right) /\left(V_{1} \otimes W_{i-1}^{\prime}\right)=V_{1} \otimes\left(W_{i}^{\prime} / W_{i-1}^{\prime}\right)
$$

in (3.3) is semistable by the earlier observation, and furthermore,

$$
\mu_{g}\left(V_{1} \otimes\left(W_{i}^{\prime} / W_{i-1}^{\prime}\right)\right)=\mu_{g}\left(V_{1}\right)+\mu_{g}\left(W_{i}^{\prime} / W_{i-1}^{\prime}\right)=\mu_{g}\left(V_{1}\right)+\mu_{g}\left(V_{2}\right) .
$$

Hence $V_{1} \otimes V_{2}$ is semistable.

Corollary 3.4. Let $V$ be a flat vector bundle over $M$. Take any integer $j \in[1, \operatorname{rank}(V)]$. If $V$ is polystable, then the exterior power $\bigwedge^{j} V$ equipped with the induced flat connection is polystable. If $V$ is semistable, then $\bigwedge^{j} V$ equipped with the induced flat connection is semistable.

Proof. If $V$ is polystable, then from Corollary 3.2 it follows that $V^{\otimes j}$ equipped with the induced flat connection is polystable. Since the flat vector bundle $\wedge^{j} V$ is a direct summand of the flat vector bundle $V^{\otimes j}$, we conclude that $\bigwedge^{j} V$ is polystable if $V^{\otimes j}$ is so.

If $V$ is semistable, then from Proposition 3.3 it follows that $V^{\otimes j}$ equipped with the induced flat connection is semistable. Therefore, the direct summand $\wedge^{j} V \subset V^{\otimes j}$ is semistable.

\section{PRINCIPAL BUNDLES ON FLAT AFFINE MANIFOLDS}

4.1. Preliminaries. Let $H$ be a Lie group. A principal $H$-bundle on $M$ is a triple of the form $\left(E_{H}, p, \psi\right)$, where $E_{H}$ is a $C^{\infty}$ manifold, $p: E_{H} \longrightarrow M$ is a $C^{\infty}$ surjective submersion, and

$$
\psi: E_{H} \times H \longrightarrow E_{H}
$$

is a smooth action of $H$ on $E_{H}$, such that

(1) $p \circ \psi=p \circ p_{1}$, where $p_{1}$ is the natural projection of $E_{H} \times H$ to $E_{H}$, and

(2) for each point $x \in M$, there is an open neighborhood $U \subset M$ of $x$, and a smooth diffeomorphism

$$
\phi: p^{-1}(U) \longrightarrow U \times H,
$$

such that $\phi$ commutes with the actions of $H$ (the group $H$ acts on $U \times H$ through right translations of $H$ ), and $q_{1} \circ \phi=p$, where $q_{1}$ is the natural projection of $U \times H$ to $U$.

Let $d p: T E_{H} \longrightarrow p^{*} T M$ be the differential of the projection $p$. A flat connection on $E_{H}$ is a $C^{\infty}$ homomorphism

$$
D: p^{*} T M \longrightarrow T E_{H}
$$

such that

- $d p \circ D=\operatorname{Id}_{p^{*} T M}$,

- the distribution $D\left(p^{*} T M\right) \subset T E_{H}$ is integrable, and 
- $D\left(p^{*} T M\right)$ is invariant under the action of $H$ on $T E_{H}$ given by the action of $H$ on $E_{H}$.

Let $H^{\prime} \subset H$ be a closed subgroup. A reduction of structure group of a principal $H-$ bundle $E_{H}$ to $H^{\prime}$ is a principal $H^{\prime}$-bundle $E_{H^{\prime}} \subset E_{H}$; the action of $H^{\prime}$ on $E_{H^{\prime}}$ is the restriction of the action of $H$ on $E_{H}$. A reduction of structure group of $E_{H}$ to $H^{\prime}$ is given by a smooth section of the fiber bundle $E_{H} / H^{\prime} \longrightarrow M$. We note that if a reduction $E_{H^{\prime}} \subset E_{H}$ corresponds to a section $\sigma$, then $E_{H}^{\prime}$ is the inverse image of $\sigma(M)$ for the quotient map $E_{H} \longrightarrow E_{H} / H^{\prime}$.

Let $D$ be a flat connection on $E_{H}$. A reduction of structure group $E_{H^{\prime}} \subset E_{H}$ to $H^{\prime}$ is said to be compatible with $D$ if for each point $z \in E_{H^{\prime}}$, the subspace $D\left(T_{p(z)} M\right) \subset T_{z} E_{H}$ is contained in the subspace $T_{z} E_{H^{\prime}} \subset T_{z} E_{H}$. Note that this condition ensures that $D$ produces a flat connection on $E_{H^{\prime}}$.

Consider the adjoint action of $H$ on its Lie algebra $\operatorname{Lie}(H)$. Let

$$
\operatorname{ad}\left(E_{H}\right):=E_{H} \times{ }^{H} \operatorname{Lie}(H) \longrightarrow M
$$

be the vector bundle over $M$ associated to the principal $H$-bundle $E_{H}$ for this action; it is known as the adjoint vector bundle for $E_{H}$. Since the adjoint action of $H$ on $\operatorname{Lie}(H)$ preserves the Lie algebra structure, the fibers of $\operatorname{ad}\left(E_{H}\right)$ are Lie algebras isomorphic to $\operatorname{Lie}(H)$. The connection $D$ on $E_{H}$ induces a connection on every fiber bundle associated to $E_{H}$. In particular, $D$ induces a connection on the vector bundle ad $\left(E_{H}\right)$; this induced connection on $\operatorname{ad}\left(E_{H}\right)$ will be denoted by $D^{\text {ad }}$. The connection $D^{\text {ad }}$ is compatible with the Lie algebra structure of the fibers of $\operatorname{ad}\left(E_{H}\right)$, meaning

$$
D^{\mathrm{ad}}([s, t])=\left[D^{\mathrm{ad}}(s), t\right]+\left[s, D^{\mathrm{ad}}(t)\right]
$$

for all locally defined smooth sections $s$ and $t$ of $\operatorname{ad}\left(E_{H}\right)$.

4.2. Stable and semistable principal bundles. Let $G_{\mathbb{C}}$ be a complex reductive linear algebraic group. A real form on $G_{\mathbb{C}}$ is an anti-holomorphic involution

$$
\sigma_{G_{\mathbb{C}}}: G_{\mathbb{C}} \longrightarrow G_{\mathbb{C}} .
$$

The real form $\sigma_{G_{\mathbb{C}}}$ is said to be of split type if there is a maximal torus $T \subset G_{\mathbb{C}}$ such that $\sigma_{G_{\mathbb{C}}}(T)=T$ and the fixed point locus of the involution $\left.\sigma_{G_{\mathbb{C}}}\right|_{T}$ of $T$ is a product of copies of $\mathbb{R}^{*}$ (the group of nonzero real numbers).

Let $G$ be a connected Lie group such that either it is a complex reductive linear algebraic group or it is the fixed point locus of a split real form $\sigma_{G_{\mathbb{C}}} \in \operatorname{Aut}\left(G_{\mathbb{C}}\right)$, where $G_{\mathbb{C}}$ and $\sigma_{G_{\mathbb{C}}}$ are as above.

If $G$ is a complex reductive group, a connected closed algebraic subgroup $P \subset G$ is called a parabolic subgroup if the quotient variety $G / P$ is complete. So, in particular, $G$ itself is a parabolic subgroup. Let $P$ be a parabolic subgroup of $G$. A character $\chi$ of $P$ is called strictly anti-dominant if the following two conditions hold:

- the line bundle over $G / P$ associated to the principal $P$-bundle $G \longrightarrow G / P$ for $\chi$ is ample, and 
- the character $\chi$ is trivial on the connected component of the center of $P$ containing the identity element.

Let $R_{u}(P) \subset P$ be the unipotent radical. The group $P / R_{u}(P)$ is called the Levi quotient of $P$. A Levi subgroup of $P$ is a connected reductive subgroup $L(P) \subset P$ such that the composition

$$
L(P) \longrightarrow P \longrightarrow P / R_{u}(P)
$$

is an isomorphism. A Levi subgroup always exists (see [Bo, page 158, $\S 11.22]$ and $[\mathrm{Hu}$, page $184, \S 30.2])$.

If $G$ is the fixed point locus of a real form $\left(G_{\mathbb{C}}, \sigma_{G_{\mathbb{C}}}\right)$, by a parabolic subgroup of $G$ we will mean a subgroup $P \subset G$ such that there is a parabolic subgroup $P^{\prime} \subset G_{\mathbb{C}}$ satisfying the conditions that $\sigma_{G_{\mathbb{C}}}\left(P^{\prime}\right)=P^{\prime}$ and $P^{\prime} \cap G=P$. By a Levi subgroup of the parabolic subgroup $P$ we will mean a subgroup $L(P) \subset P$ such that there is a Levi subgroup $L\left(P^{\prime}\right) \subset P^{\prime}$ satisfying the conditions that $\sigma_{G_{\mathbb{C}}}\left(L\left(P^{\prime}\right)\right)=L\left(P^{\prime}\right)$ and $L\left(P^{\prime}\right) \bigcap G=L(P)$.

Let $\left(E_{G}, D\right)$ be a flat principal $G$-bundle over $M$.

It is called semistable (respectively, stable) if for every triple of the form $\left(Q, E_{Q}, \lambda\right)$, where $Q \subset G$ is a proper parabolic subgroup, and $E_{Q} \subset E_{G}$ is a reduction of structure group of $E_{G}$ to $Q$ compatible with $D$, and $\lambda$ is a strictly anti-dominant character of $Q$, the inequality

$$
\operatorname{deg}_{g}\left(E_{Q}(\lambda)\right) \geq 0
$$

(respectively, $\operatorname{deg}_{g}\left(E_{Q}(\lambda)\right)>0$ ) holds, where $E_{Q}(\lambda)$ is the flat line bundle over $M$ associated to the flat principal $Q$-bundle $E_{Q}$ for the character $\lambda$ of $Q$.

In order to decide whether $\left(E_{G}, D\right)$ is semistable (respectively, stable), it suffices to verify the above inequality (respectively, strict inequality) only for those $Q$ which are proper maximal parabolic subgroups of $G$. More precisely, $E_{G}$ is semistable (respectively, stable) if and only if for every pair $(Q, \sigma)$, where $Q \subset G$ is a proper maximal parabolic subgroup, and $\sigma: M \longrightarrow E_{G} / Q$ is a reduction of structure group of $E_{G}$ to $Q$ compatible with $D$, the inequality

$$
\operatorname{deg}_{g}\left(\sigma^{*} T_{\text {rel }}\right) \geq 0
$$

(respectively, $\operatorname{deg}_{g}\left(\sigma^{*} T_{\text {rel }}\right)>0$ ) holds, where $T_{\text {rel }}$ is the relative tangent bundle over $E_{G} / Q$ for the projection $E_{G} / Q \longrightarrow M$. (See [Ra, page 129, Definition 1.1] and [Ra, page 131, Lemma 2.1].) It should be mentioned that the connection $D$ on $E_{G}$ induces a flat connection on the associated fiber bundle $E_{G} / Q \longrightarrow M$. Since the section $\sigma$ is flat with respect to this induced connection (it is flat because the reduction $E_{Q}$ is compatible with $D)$, the pullback $\sigma^{*} T_{\text {rel }}$ gets a flat connection.

Let $\left(E_{G}, D\right)$ be a flat principal $G$-bundle over $M$. A reduction of structure group

$$
E_{Q} \subset E_{G}
$$

to a parabolic subgroup $Q \subset G$ compatible with $D$ is called admissible if for each character $\lambda$ of $Q$ trivial on the center of $G$, the associated flat line bundle $E_{Q}(\lambda) \longrightarrow M$ 
satisfies the following condition:

$$
\operatorname{deg}_{g}\left(E_{Q}(\lambda)\right)=0
$$

We will call $\left(E_{G}, D\right)$ to be polystable if either $E_{G}$ is stable, or there is a proper parabolic subgroup $Q$ and a reduction of structure group $E_{L(Q)} \subset E_{H}$ to a Levi subgroup $L(Q)$ of $Q$ compatible with $D$ such that the flat principal $L(Q)$-bundle $E_{L(Q)}$ is stable, and the reduction of structure group of $E_{G}$ to $Q$, obtained by extending the structure group of $E_{L(Q)}$ using the inclusion of $L(Q)$ in $Q$, is admissible.

We note that a flat polystable principal $G$-bundle on $M$ is semistable.

For notational convenience, we will omit the symbol of connection for a flat principal bundle. When we will say " $E_{G}$ be a flat principal $G$-bundle" it will mean that $E_{G}$ is equipped with a flat connection.

4.3. Harder-Narasimhan reduction of principal bundles. Let $G$ be as before. Let $E_{G}$ be a flat principal $G$-bundle over $M$.

A Harder-Narasimhan reduction of $E_{G}$ is a pair of the form $\left(P, E_{P}\right)$, where $P \subset G$ is a parabolic subgroup, and $E_{P} \subset E_{G}$ is a reduction of structure group of $E_{G}$ to $P$ compatible with the connection such that the following two conditions hold:

(1) The principal $P / R_{u}(P)$-bundle $E_{P} / R_{u}(P)$ equipped with the induced flat connection is semistable, where $R_{u}(P) \subset P$ is the unipotent radical.

(2) For any nontrivial character $\chi$ of $P$ which can be expressed as a nonnegative integral combination of simple roots, the flat line bundle over $M$ associated to $E_{P}$ for $\chi$ is of positive degree.

Proposition 4.1. A flat principal $G$-bundle $E_{G}$ admits a Harder-Narasimhan reduction. If $\left(P, E_{P}\right)$ and $\left(P, E_{P}\right)$ are two Harder-Narasimhan reductions of $E_{G}$, then there is an element $g \in G$ such that $Q=g^{-1} P g$ and $E_{Q}=E_{P} g$.

Proof. Let $\operatorname{ad}\left(E_{G}\right) \longrightarrow M$ be the adjoint vector bundle of $E_{G}$ (defined in (4.1)). As mentioned earlier, the flat connection on $E_{G}$ induces a flat connection on $\operatorname{ad}\left(E_{G}\right)$. Let

$$
0=V_{0} \subset V_{1} \subset \cdots \subset V_{\ell-1} \subset V_{\ell}=\operatorname{ad}\left(E_{G}\right)
$$

be the Harder-Narasimhan filtration of the flat vector bundle $\operatorname{ad}\left(E_{G}\right)$ constructed in Theorem 2.5. Using Proposition 3.3 it can be deduced that $\ell$ in (4.5) is an odd integer; its proof is identical to the proof of (3) in [AB, p. 215]. The flat subbundle

$$
V_{(\ell+1) / 2} \subset \operatorname{ad}\left(E_{G}\right)
$$

in (4.5) is the adjoint vector bundle of a reduction of structure group of $E_{G}$ to a parabolic subgroup; its proof is identical to the proof of [AAB, p. 699, Lemma 4]. After we fix a parabolic subgroup $P$ of $G$ in the conjugacy class of parabolic subgroups of $G$ defined by the subalgebra $\left(E_{(\ell+1) / 2}\right)_{x} \subset \operatorname{ad}\left(E_{G}\right)_{x}$, where $x \in M$, we get a reduction of structure group of $E_{G}$ to $P$ compatible with the connection. This reduction satisfies all the conditions in the proposition. The details of the argument are in $\mathrm{AAB}$. 
The second condition in the Harder-Narasimhan reduction can be reformulated in other equivalent ways; see $\mathrm{AAB}]$.

Remark 4.2. Proposition 4.1 can also be proved by imitating the proof of Proposition 3.1 in $[\mathrm{BH}]$.

Proposition 4.1 has the following corollary:

Corollary 4.3. A flat principal $G$-bundle $E_{G}$ over $M$ is semistable if and only if the flat vector bundle $\operatorname{ad}\left(E_{G}\right)$ is semistable.

Proof. Assume that ad $\left(E_{G}\right)$ is not semistable. Then $E_{(\ell+1) / 2}$ in (4.5) is a proper subbundle of $\operatorname{ad}\left(E_{G}\right)$. Hence $E_{G}$ has a nontrivial Harder-Narasimhan reduction $\left(P, E_{P}\right)$. Let $\mathfrak{g}$ and $\mathfrak{p}$ be the Lie algebras of $G$ and $P$ respectively. The group $P$ has the adjoint action on $\mathfrak{g} / \mathfrak{p}$. The vector bundle over $M$ associated to the principal $P$-bundle $E_{P}$ for the $P-$ module $\mathfrak{g} / \mathfrak{p}$ is identified with the vector bundle $\operatorname{ad}\left(E_{G}\right) / E_{(\ell+1) / 2}$. Consequently, the reduction $E_{P} \subset E_{G}$ and the strictly anti-dominant character of $P$ defined by the $P-$ module $\Lambda^{\text {top }}(\mathfrak{g} / \mathfrak{p})$ violate the inequality in (4.2). Hence the flat principal $G$-bundle $E_{G}$ is not semistable.

To prove the converse, assume that the flat vector bundle $\operatorname{ad}\left(E_{G}\right)$ is semistable. Then $E_{(\ell+1) / 2}=\operatorname{ad}\left(E_{G}\right)$ (see (4.6) $)$. Hence the Harder-Narasimhan reduction of $E_{G}$ is $\left(G, E_{G}\right)$ itself. Since the Levi quotient of $G$ is $G$ itself, from the first condition in the definition of a Harder-Narasimhan reduction we conclude that $E_{G}$ is semistable.

Corollary 4.4. Assume that $G$ is the fixed point locus of a split real form on $G_{\mathbb{C}}$. Let $E_{G}$ be a flat principal $G$-bundle over $M$. Let $E_{G_{\mathbb{C}}}$ be the flat principal $G_{\mathbb{C}}$-bundle over $M$ obtained by extending the structure group of $E_{G}$ using the inclusion of $G$ in $G_{\mathbb{C}}$. The principal $G$-bundle $E_{G}$ is semistable if and only if the principal $G_{\mathbb{C}}$-bundle $E_{G_{\mathbb{C}}}$ is so.

Proof. Let $V$ be a flat real vector bundle over $M$. Let $V_{\mathbb{C}}:=V \bigotimes_{\mathbb{R}} \mathbb{C}$ be the flat complex vector bundle. We will show that $V$ is semistable if and only if $V_{\mathbb{C}}$ is so.

If a flat subbundle $W \subset V$ violates the semistability condition for $V$, then the flat subbundle $W \bigotimes_{\mathbb{R}} \mathbb{C} \subset V_{\mathbb{C}}$ violates the semistability condition for $V_{\mathbb{C}}$. Therefore, $V$ is semistable if $V_{\mathbb{C}}$ is so.

To prove the converse, assume that $V_{\mathbb{C}}$ is not semistable. Let $F \subset V_{\mathbb{C}}$ be the maximal semistable subbundle of $V_{\mathbb{C}}$, which is a proper subbundle because $V_{\mathbb{C}}$ is not semistable. From the uniqueness of $F$ it follows immediately that the $\mathbb{R}$-linear conjugation automorphism of $V_{\mathbb{C}}=V \bigotimes_{\mathbb{R}} \mathbb{C}$ defined by $v \otimes \lambda \longmapsto v \otimes \bar{\lambda}$, where $v \in V$ and $\lambda \in \mathbb{C}$, preserves the subbundle $F$. Hence $F$ is the complexification of a flat subbundle $F^{\prime}$ of $V$. This subbundle $F^{\prime}$ violates the semistability condition for $V$. Therefore, $V_{\mathbb{C}}$ is semistable if and only if $V$ is so.

We apply the above observation to $V=\operatorname{ad}\left(E_{G}\right)$. Note that

$$
\operatorname{ad}\left(E_{G_{\mathbb{C}}}\right)=\operatorname{ad}\left(E_{G}\right) \otimes_{\mathbb{R}} \mathbb{C} .
$$

In view of Corollary 4.3, the proof is complete. 


\section{THE SOCLE REDUCTION}

Let $V \longrightarrow M$ be a flat semistable vector bundle; it is allowed to be real or complex. Let $F_{1}$ and $F_{2}$ be two flat subbundles of $V$ such that both $F_{1}$ and $F_{2}$ are polystable, and

$$
\mu_{g}\left(F_{1}\right)=\mu_{g}\left(F_{2}\right)=\mu_{g}(V) .
$$

Let

$$
F_{1}+F_{2} \subset V
$$

be the flat subbundle of $V$ generated by $F_{1}$ and $F_{2}$.

Proposition 5.1. The flat vector bundle $F_{1}+F_{2}$ is polystable, and $\mu_{g}\left(F_{1}+F_{2}\right)=\mu_{g}(V)$.

Proof. From (5.1),

$$
\mu_{g}\left(F_{1} \oplus F_{2}\right)=\mu_{g}(V) .
$$

Consider the short exact sequence of flat vector bundles

$$
0 \longrightarrow F_{1} \cap F_{2} \longrightarrow F_{1} \oplus F_{2} \longrightarrow F_{1}+F_{2} \longrightarrow 0 \text {. }
$$

If $F_{1} \cap F_{2}=0$, then $F_{1}+F_{2}=F_{1} \oplus F_{2}$, hence in this case $F_{1}+F_{2}$ is polystable, and $\mu_{g}\left(F_{1}+F_{2}\right)=\mu_{g}(V)$ from (5.2). Therefore, the proposition is evident if $F_{1} \cap F_{2}=0$.

So assume that $\operatorname{rank}\left(F_{1} \cap F_{2}\right)>0$.

Since $V$ is semistable, and both $F_{1} \cap F_{2}$ and $F_{1}+F_{2}$ are flat subbundles of $V$, we have

$$
\mu_{g}\left(F_{1} \cap F_{2}\right), \mu_{g}\left(F_{1}+F_{2}\right) \leq \mu_{g}(V) .
$$

From (5.3),

$$
\mu_{g}\left(F_{1} \oplus F_{2}\right)=\frac{\mu_{g}\left(F_{1} \cap F_{2}\right) \cdot \operatorname{rank}\left(F_{1} \cap F_{2}\right)+\mu_{g}\left(F_{1}+F_{2}\right) \cdot \operatorname{rank}\left(F_{1}+F_{2}\right)}{\operatorname{rank}\left(F_{1} \cap F_{2}\right)+\operatorname{rank}\left(F_{1}+F_{2}\right)} .
$$

Combining this with (5.2) and (5.4),

$$
\mu_{g}\left(F_{1}+F_{2}\right)=\mu_{g}\left(F_{1} \cap F_{2}\right)=\mu_{g}(V) .
$$

Let $r$ be the rank of the flat vector bundle $F_{1} \cap F_{2}$; recall that it is positive. Consider the vector bundle

$$
\mathcal{W}:=\mathcal{H o m}\left(\bigwedge^{r}\left(F_{1} \cap F_{2}\right), \bigwedge^{r} F_{1}\right)=\bigwedge^{r}\left(F_{1} \cap F_{2}\right)^{*} \otimes \bigwedge^{r} F_{1} .
$$

Note that the inclusion homomorphism $F_{1} \cap F_{2} \hookrightarrow F_{1}$ defines a nonzero flat section

$$
\eta \in H^{0}(M, \mathcal{W}) \text {. }
$$

We have

$$
\mu_{g}(\mathcal{W})=\mu_{g}\left(\bigwedge^{r} F_{1}\right)-\mu_{g}\left(\bigwedge^{r}\left(F_{1} \cap F_{2}\right)\right)=r \cdot \mu_{g}\left(F_{1}\right)-r \cdot \mu_{g}\left(F_{1} \cap F_{2}\right) .
$$

Hence $\mu_{g}(\mathcal{W})=0$ by (5.1) and (5.5). Hence, $\operatorname{deg}_{g}(\mathcal{W})=0$; also, from Corollary 3.4 we know that $\mathcal{W}$ is polystable (note that $\bigwedge^{r}\left(F_{1} \cap F_{2}\right)^{*}$ is a line bundle). We recall from [Lo] that given any flat vector bundle $V$ on $M$ of degree zero equipped with a HermitianEinstein connection $\nabla_{V}$, any flat section of $V$ is flat with respect to $\nabla_{V}$ (see Theorem 3 of [Lo, p. 110]). Also, [Lo, p. 102, Theorem 1] and [Lo, p. 129, Corollary 33] say that 
any polystable vector bundle on $M$ admits a Hermitian-Einstein connection. Hence the vector bundle $\mathcal{W}$ in (5.6) admits a Hermitian-Einstein connection, and the section $\eta$ in (5.7) is flat with respect to the Hermitian-Einstein connection on $\mathcal{W}$.

Since $\eta$ is flat with respect to the Hermitian-Einstein connection on $\mathcal{W}$, it follows that the Hermitian-Einstein connection on $F_{1}$ preserves the subbundle $F_{1} \cap F_{2} \subset F_{1}$. Consequently, $F_{1} \cap F_{2}$ is polystable [Lo, p. 110, Theorem 4]. This also implies that the orthogonal complement of $F_{1} \cap F_{2}$ with respect to a Hermitian-Einstein metric on $F_{1}$

$$
F^{\prime}:=\left(F_{1} \cap F_{2}\right)^{\perp} \subset F_{1}
$$

is preserved by the Hermitian-Einstein connection. Hence $F^{\prime}$ is polystable if $F^{\prime} \neq 0$; note that $\mu_{g}\left(F^{\prime}\right)=\mu_{g}\left(F_{1}\right)$ if $F^{\prime} \neq 0$.

Since $F_{1}+F_{2}=F^{\prime} \oplus F_{2}$, we now conclude that $F_{1}+F_{2}$ is polystable, and $\mu_{g}\left(F_{1}+F_{2}\right)=$ $\mu_{g}(V)$.

Corollary 5.2. Let $V \longrightarrow M$ be a flat semistable vector bundle. Then there is a unique maximal polystable flat subbundle $F \subset V$ such that $\mu_{g}(F)=\mu_{g}(V)$.

Proof. In view of Proposition 5.1, the flat subbundle $F \subset V$ generated by all flat polystable subbundles $E \subset V$ with $\mu_{g}(E)=\mu_{g}(V)$ satisfies the conditions in the corollary.

The flat polystable subbundle $F \subset V$ in Corollary 5.2 is called the socle of $V$.

If $F$ is properly contained in $V$, then we note that $V / F$ is semistable, and $\mu_{g}(V / F)=$ $\mu_{g}(V)$. Therefore, Corollary 5.2 gives the following:

Corollary 5.3. Let $V \longrightarrow M$ be a flat semistable vector bundle. Then there is a filtration of flat subbundles

$$
0=F_{0} \subset F_{1} \subset \cdots \subset F_{n-1} \subset F_{n}=V
$$

such that for each $i \in[1, n]$, the flat subbundle $F_{i} / F_{i-1} \subset V / F_{i-1}$ is the socle of the flat semistable vector bundle $V / F_{i-1}$.

5.1. Socle reduction of a principal bundle. Let $G$ be as before. Let $E_{G} \longrightarrow M$ be a semistable principal $G$-bundle.

A socle reduction of $E_{G}$ is a pair $\left(Q_{0}, E_{Q_{0}}\right)$, where

- $Q_{0} \subset H$ is maximal among all the parabolic subgroups $Q$ of $G$ such that $E_{G}$ admits an admissible reduction of structure group

$$
E_{Q} \subset E_{G}
$$

for which the corresponding principal $Q / R_{u}(Q)$-bundle $E_{Q} / R_{u}(Q) \longrightarrow M$ is polystable, where $R_{u}(Q)$ is the unipotent radical of $Q$, and

- $E_{Q_{0}} \subset E_{G}$ is an admissible reduction of structure group of $E_{G}$ to $Q_{0}$ such that the associated principal $Q_{0} / R_{u}\left(Q_{0}\right)$-bundle $E_{Q_{0}} / R_{u}\left(Q_{0}\right)$ is polystable.

(Admissible reductions were defined in (4.4).) 
Proposition 5.4. Let $E_{G} \longrightarrow M$ be a semistable principal $G$-bundle. Then $E_{G}$ admits a socle reduction. If $\left(Q_{1}, E_{Q_{1}}\right)$ and $\left(Q_{2}, E_{Q_{2}}\right)$ are two socle reductions of $E_{G}$, then there is an element $g \in G$ such that $Q_{2}=g^{-1} Q_{1} g$ and $E_{Q_{2}}=E_{Q_{1}} g$.

Proof. From Corollary 4.3 we know that the flat adjoint bundle $\operatorname{ad}\left(E_{G}\right)$ is semistable. Let

$$
0=F_{0} \subset F_{1} \subset \cdots \subset F_{n-1} \subset F_{n}=\operatorname{ad}\left(E_{G}\right)
$$

be the filtration constructed in Corollary 5.3. Using Corollary 3.2 it can be shown that $n$ is an odd integer; see [AB, p. 218] for the details. The flat subbundle

$$
F_{(n+1) / 2} \subset \operatorname{ad}\left(E_{G}\right)
$$

in (5.8) is the adjoint vector bundle of a reduction of structure group of $E_{G}$ to a parabolic subgroup. After we fix a parabolic subgroup $Q_{0}$ of $G$ in the conjugacy class of parabolic subgroups of $G$ defined by the subalgebra $\left(E_{(n+1) / 2}\right)_{x} \subset \operatorname{ad}\left(E_{G}\right)_{x}$, where $x \in M$, we get a reduction of structure group $E_{Q_{0}} \subset E_{G}$ to $Q_{0}$ compatible with the connection on $E_{G}$. It can be shown that this pair $\left(Q_{0}, E_{Q_{0}}\right)$ is a socle reduction of $E_{G}$ [AB. The uniqueness statement is also proved in $\mathrm{AB}$.

From Proposition 5.4 and its proof we have the following corollary:

Corollary 5.5. Let $E_{G}$ be a flat principal $G$-bundle over $M$. Then $E_{G}$ is polystable if and only if the flat vector bundle $\operatorname{ad}\left(E_{G}\right)$ is polystable.

Proof. First we assume that $E_{G}$ is polystable. Then the flat vector bundle $\operatorname{ad}\left(E_{G}\right)$ is semistable by Corollary 4.3. If $\operatorname{ad}\left(E_{G}\right)$ is not polystable, and $\left(Q_{0}, E_{Q_{0}}\right)$ is a socle reduction of $E_{G}$, then $Q_{0}$ is a proper parabolic subgroup of $G$. Therefore, $E_{G}$ is not polystable, which contradicts the assumption. Hence $\operatorname{ad}\left(E_{G}\right)$ is polystable.

To prove the converse, assume that $\operatorname{ad}\left(E_{G}\right)$ is polystable. Then the principal $G$-bundle $E_{G}$ is semistable (see Corollary 4.3). Consider the socle of $E_{G}$. Since $\operatorname{ad}\left(E_{G}\right)$ is polystable, we have $E_{(n+1) / 2}=\operatorname{ad}\left(E_{G}\right)$ (see (5.9) ). Hence $\left(G, E_{G}\right)$ is the socle of $E_{G}$. Therefore, from the definition of a socle we conclude that $E_{G}$ is polystable.

Corollary 5.6. Assume that $G$ is the fixed point locus of a split real form on $G_{\mathbb{C}}$. Let $E_{G}$ be a flat principal $G$-bundle over $M$. Let $E_{G_{\mathbb{C}}}$ be the flat principal $G_{\mathbb{C}}$-bundle over $M$ obtained by extending the structure group of $E_{G}$ using the inclusion of $G$ in $G_{\mathbb{C}}$. The principal $G$-bundle $E_{G}$ is polystable if and only if the principal $G_{\mathbb{C}}$-bundle $E_{G_{\mathbb{C}}}$ is so.

Proof. We note that $E_{G}$ is polystable if and only if $\operatorname{ad}\left(E_{G}\right)$ is polystable by Corollary 5.5 . Since

$$
\operatorname{ad}\left(E_{G_{\mathbb{C}}}\right)=\operatorname{ad}\left(E_{G}\right) \otimes_{\mathbb{R}} \mathbb{C}=\operatorname{ad}\left(E_{G}\right) \oplus \sqrt{-1} \cdot \operatorname{ad}\left(E_{G}\right),
$$

it follows that $\operatorname{ad}\left(E_{G}\right)$ is polystable if and only if $\operatorname{ad}\left(E_{G_{\mathbb{C}}}\right)$ is polystable. From Corollary 5.5, the adjoint vector bundle $\operatorname{ad}\left(E_{G_{\mathbb{C}}}\right)$ is polystable if and only if $E_{G_{\mathbb{C}}}$ is polystable. 


\section{Hermitian-Einstein connection on stable Bundles and Bogomolov INEQUALITY}

6.1. Hermitian-Einstein connection and stable principal bundles. Fix a maximal compact subgroup

$$
K \subset G
$$

of the reductive group $G$. Let $E_{G}$ be a flat principal $G$-bundle over $M$. A Hermitian structure on $E_{G}$ is a $C^{\infty}$ reduction of structure group

$$
E_{K} \subset E_{G} .
$$

Recall that $G$ is either the fixed point locus of a split real form on a complex reductive group $G_{\mathbb{C}}$ or $G$ is complex reductive. In the second case, by $G_{\mathbb{C}}$ we will denote $G$ itself; this is for notational convenience.

Given a flat principal $G$-bundle $E_{G}$ over $M$, we get a holomorphic principal $G_{\mathbb{C}}$-bundle $E_{G_{\mathbb{C}}}$ over $M_{\mathbb{C}}$ (the total space of $T M$ ); see [Lo, p. 102].

Given a Hermitian structure on $E_{G}$, there is a naturally associated connection on the principal $G_{\mathbb{C}}$-bundle $E_{G_{\mathbb{C}}}$ over $M_{\mathbb{C}}[$ Lo, p. 106, Lemma 1]; although [Lo, Lemma 1] is only for vector bundles, the proof for principal bundles is identical.

Any element $z$ of the center of the Lie algebra $\mathfrak{g}=\operatorname{Lie}(G)$ defines a flat section of $\operatorname{ad}\left(E_{G}\right)$, because $z$ is fixed by the adjoint action of $G$ on $\mathfrak{g}$; this section of $\operatorname{ad}\left(E_{G}\right)$ given by $z$ will be denoted by $\underline{z}$.

Let $E_{K} \subset E_{G}$ be a Hermitian structure on $E_{G}$. Let $\nabla$ be the corresponding connection on $E_{G_{\mathbb{C}}}$. The curvature of $\nabla$ will be denoted by $K(\nabla)$. So $K(\nabla)$ is a smooth $(1,1)$-form on $M_{\mathbb{C}}$ with values in the adjoint vector bundle $\operatorname{ad}\left(E_{G_{\mathbb{C}}}\right)$. Contracting it using the metric $g$, we get a smooth section $\Lambda_{g} K(\nabla)$ of $\operatorname{ad}\left(E_{G}\right)$. The Hermitian structure $E_{K}$ is called Hermitian-Einstein if there is an element $z$ in the center of the Lie algebra $\mathfrak{g}$ such that

$$
\Lambda_{g} K(\nabla)=\underline{z} .
$$

If $E_{K}$ is a Hermitian-Einstein structure, then the corresponding connection $\nabla$ is called a Hermitian-Einstein connection.

Theorem 6.1. A flat principal $G$-bundle $E_{G} \longrightarrow M$ admits a Hermitian-Einstein structure if and only if $E_{G}$ is polystable. A polystable flat principal $G$-bundle admits a unique Hermitian-Einstein connection.

Proof. First we assume that $E_{G}$ admits a Hermitian-Einstein structure. A HermitianEinstein structure on $E_{G}$ induces a Hermitian-Einstein metric on the adjoint vector bundle $\operatorname{ad}\left(E_{G}\right)$. Hence $\operatorname{ad}\left(E_{G}\right)$ is polystable [Lo, p, 110, Theorem 4]. Hence $E_{G}$ is polystable by Corollary 5.5 .

To prove the converse, assume $E_{G}$ is polystable. We will first reduce to the case that $G$ is complex reductive.

If $G$ is the fixed point locus of a split real form on $G_{\mathbb{C}}$, then Corollary 5.6 says that the corresponding principal $G_{\mathbb{C}}$-bundle $E_{G_{\mathbb{C}}}$ is also polystable. In the following paragraphs, 
we produce a unique Hermitian-Einstein connection on $E_{G_{\mathbb{C}}}$. Uniqueness implies that it is invariant under a natural complex conjugation, as in the proof of Corollary 4.4 above, and so the Hermitian-Einstein connection on $E_{G_{\mathbb{C}}}$ reduces to a connection on $E_{G}$.

We assume that $G$ is complex reductive. Let $Z_{G} \subset G$ be the center of $G$. The adjoint action of $G / Z_{G}$ on $\mathfrak{g}$ is faithful. Since $G$ is reductive, the quotient $G /[G, G]$ is a product of copies of $\mathbb{C}^{*}$.

Let $E_{G} \longrightarrow M$ be a flat polystable principal $G$-bundle. Then the vector bundle $\operatorname{ad}\left(E_{G}\right)$ is polystable by Corollary 5.5. Let $\nabla(\mathrm{ad})$ be the Hermitian-Einstein connection on $\operatorname{ad}\left(E_{G}\right)$.

We will show that the connection $\nabla($ ad) preserves the Lie algebra structure of the fibers of $\operatorname{ad}\left(E_{G}\right)$.

Let

$$
\theta_{0}: \operatorname{ad}\left(E_{G}\right) \otimes \operatorname{ad}\left(E_{G}\right) \longrightarrow \operatorname{ad}\left(E_{G}\right)
$$

be the homomorphism defined by the Lie algebra structure of the fibers of $\operatorname{ad}\left(E_{G}\right)$. Define the flat vector bundle

$$
\mathcal{W}:=\mathcal{H} o m\left(\operatorname{ad}\left(E_{G}\right) \otimes \operatorname{ad}\left(E_{G}\right), \operatorname{ad}\left(E_{G}\right)\right)=\left(\operatorname{ad}\left(E_{G}\right) \otimes \operatorname{ad}\left(E_{G}\right)\right)^{*} \otimes \operatorname{ad}\left(E_{G}\right) .
$$

Let

$$
\theta \in C^{\infty}(M, \mathcal{W})
$$

be the smooth section defined by the above homomorphism $\theta_{0}$. We note that $\theta$ is flat with respect to the flat connection on $\mathcal{W}$ induced by the flat connection on $E_{G}$.

Fix a nondegenerate $G$-invariant symmetric bilinear form $B$ on $\mathfrak{g}$; such a form exists because $G$ is either complex reductive or the fixed point locus of a real form of a complex reductive group. Since $B$ is $G$-invariant, it produces a symmetric bilinear form $\widetilde{B}$ on $\operatorname{ad}\left(E_{G}\right)$ which is fiberwise nondegenerate and is preserved by the flat connection on $\operatorname{ad}\left(E_{G}\right)$. Therefore, $\widetilde{B}$ produces an isomorphism of the flat vector bundle $\operatorname{ad}\left(E_{G}\right)$ with its dual $\operatorname{ad}\left(E_{G}\right)^{*}$. Hence $\operatorname{deg}_{g}\left(\operatorname{ad}\left(E_{G}\right)\right)=-\operatorname{deg}_{g}\left(\operatorname{ad}\left(E_{G}\right)^{*}\right)=-\operatorname{deg}_{g}\left(\operatorname{ad}\left(E_{G}\right)\right)$, implying that

$$
\operatorname{deg}_{g}\left(\operatorname{ad}\left(E_{G}\right)\right)=0 .
$$

Hence

$$
\operatorname{deg}_{g}(\mathcal{W})=0
$$

where $\mathcal{W}$ is defined in (6.1).

The Hermitian-Einstein connection $\nabla(\mathrm{ad})$ on $\operatorname{ad}\left(E_{G}\right)$ induces a connection on the vector bundle $\mathcal{W}$; this induced connection will be denoted by $\widetilde{\nabla}$. Since the connection $\nabla(\operatorname{ad})$ is Hermitian-Einstein, the connection $\widetilde{\nabla}$ is also Hermitian-Einstein. Therefore, from (6.3) it follows that any flat section of $\mathcal{W}$ is also flat with respect to the Hermitian-Einstein connection $\widetilde{\nabla}$ [Lo, p. 110, Theorem 3]. In particular, the section $\theta$ in (6.2) is flat with respect to $\widetilde{\nabla}$. This immediately implies that the connection $\nabla(\mathrm{ad})$ preserves the Lie algebra structure of the fibers of $\operatorname{ad}\left(E_{G}\right)$. 
Hence the connection $\nabla(\mathrm{ad})$ produces a connection on the flat principal $G / Z_{G}$-bundle $E_{G} / Z_{G}$. This connection on $E_{G} / Z_{G}$ will be denoted by $\nabla^{\prime}$. The connection $\nabla^{\prime}$ is Hermitian-Einstein because $\nabla(\mathrm{ad})$ is so.

Since $G /[G, G]=\left(\mathbb{C}^{*}\right)^{d}$, where $d$ is the dimension of $Z_{G}$, the flat principal $G /[G, G]_{-}$ bundle $E_{G} /[G, G]$ admits a unique Hermitian-Einstein connection; this connection will be denoted by $\nabla^{\prime \prime}$.

The quotient map $G \longrightarrow\left(G / Z_{G}\right) \times(G /[G, G])$ has the property that the corresponding homomorphism of Lie algebras is an isomorphism. Therefore, there is a natural bijection between the connections on a principal $G$-bundle $F_{G}$ and the connections on the principal $\left.G / Z_{G}\right) \times(G /[G, G])$-bundle obtained by extending the structure group of $F_{G}$ using the above homomorphism $G \longrightarrow\left(G / Z_{G}\right) \times(G /[G, G])$. The connections $\nabla^{\prime}$ and $\nabla^{\prime \prime}$ on $E_{G} / Z_{G}$ and $E_{G} /[G, G]$ together define a connection on the principal $\left.G / Z_{G}\right) \times(G /[G, G])-$ bundle $\left(E_{G} / Z_{G}\right) \times_{M}\left(E_{G} /[G, G]\right)$ over $M$. By the above remark on bijection of connections, this connection on $\left(E_{G} / Z_{G}\right) \times_{M}\left(E_{G} /[G, G]\right)$ produces a connection on $E_{G}$. The connection on $E_{G}$ obtained this way is Hermitian-Einstein because both $\nabla^{\prime}$ and $\nabla^{\prime \prime}$ are so.

The uniqueness of a Hermitian-Einstein connection on $E_{G}$ follows from the uniqueness of the Hermitian-Einstein connections on the vector bundle $\operatorname{ad}\left(E_{G}\right)$ and the principal $G /[G, G]$-bundle $E_{G} /[G, G]$. This completes the proof of the theorem.

6.2. A Bogomolov type inequality. As before, $M$ is a compact connected special flat affine manifold, $g$ is a Gauduchon metric on $M$, and $\nu$ is a nonzero covariant constant volume form on $M$. Let $d$ be the dimension of $M$. The $(1,1)$-form given by $g$ will be denoted by $\omega_{g}$. We recall that the Gauduchon condition says that

$$
\partial \bar{\partial}\left(\omega_{g}^{d-1}\right)=0
$$

(see [Lo, p. 109]).

The Gauduchon metric $g$ is called astheno-Kähler if

$$
\partial \bar{\partial}\left(\omega_{g}^{d-2}\right)=0
$$

(see [JY, p. 246]).

We note that if $d=2$, then $g$ is astheno-Kähler. If $g$ is Kähler, then $g$ is also asthenoKähler.

We assume that the Gauduchon metric $g$ is astheno-Kähler.

Let $E$ be a flat vector bundle on $M$. Take a Hermitian structure $h$ on $E$. Using (6.4) it follows that

$$
\int_{M} \frac{c_{1}(E, h)^{2} \omega_{g}^{d-2}}{\nu} \in \mathbb{R} \text { and } \int_{M} \frac{c_{2}(E, h) \omega_{g}^{d-2}}{\nu} \in \mathbb{R}
$$

are independent of the choice of $h$.

Lemma 6.2. Let $V \longrightarrow M$ be a semistable flat vector bundle of rank $r$. Then

$$
\int_{M} \frac{c_{2}(\mathcal{E} n d(V)) \omega_{g}^{d-2}}{\nu}=\int_{M} \frac{\left(2 r \cdot c_{2}(V)-(r-1) c_{1}(V)^{2}\right) \omega_{g}^{d-2}}{\nu} \geq 0 .
$$


Proof. First assume that $V$ is polystable. Therefore, $V$ admits a Hermitian-Einstein connection (see [Lo, p. 102, Theorem 1] for complex case and [Lo, p. 129, Corollary 33] for real case). Let $h$ be a Hermitian-Einstein metric on $V$. Then the $d$-form

$$
\frac{\left(2 r \cdot c_{2}(V, h)-(r-1) c_{1}(V, h)^{2}\right) \omega_{g}^{d-2}}{\nu}
$$

on $M$ is pointwise nonnegative (see [Lu] and [LYZ, p. 107] for the computation). Therefore, the lemma is proved for polystable vector bundles.

If $V$ is semistable, then there is a filtration of flat subbundles

$$
0=V_{0} \subset V_{1} \subset \cdots \subset V_{n-1} \subset V_{\ell}=V
$$

such that $V_{i} / V_{i-1}$ is polystable for all $i \in[1, n]$, and also $\mu_{g}\left(V_{i} / V_{i-1}\right)=\mu_{g}(V)$. Since the inequality in the statement of the lemma holds for all $V_{i} / V_{i-1}$, it also holds for $V$.

Let $G$ be a connected Lie group such that it is either a complex reductive linear algebraic group or it is the fixed point locus of a split real form on a complex reductive linear algebraic group

Proposition 6.3. Let $E_{G} \longrightarrow M$ be a flat semistable principal G-bundle. Then

$$
\int_{M} \frac{c_{2}\left(\operatorname{ad}\left(E_{G}\right)\right) \omega_{g}^{d-2}}{\nu} \geq 0 .
$$

Proof. The vector bundle $\operatorname{ad}\left(E_{G}\right)$ is semistable because $E_{G}$ is semistable (see Corollary 4.3). Since $\operatorname{ad}\left(E_{G}\right)=\operatorname{ad}\left(E_{G}\right)^{*}$, we have

$$
\int_{M} \frac{c_{1}\left(\operatorname{ad}\left(E_{G}\right)\right)^{2} \omega_{g}^{d-2}}{\nu}=0 .
$$

Hence the proof is completed by Lemma 6.2.

\section{REFERENCES}

[AB] B. Anchouche and I. Biswas, Einstein-Hermitian connections on polystable principal bundles over a compact Kähler manifold, Amer. Jour. Math. 123 (2001), 207-228.

[AAB] B. Anchouche, H. Azad and I. Biswas, Harder-Narasimhan reduction for principal bundles over a compact Kähler manifold, Math. Ann. 323 (2002), 693-712.

$[\mathrm{BH}] \quad$ I. Biswas and Y. I. Holla, Harder-Narasimhan reduction of a principal bundle, Nagoya Math. Jour. 174 (2004), 201-223.

[Bo] A. Borel, Linear Algebraic Groups, Second edition, Graduate Texts in Mathematics, 126, Springer-Verlag, New York, 1991.

[Br] L. Bruasse, Harder-Narasimhan filtration on non Kähler manifolds, Internat. Jour. Math. 12 (2001), 579-594.

[Hu] J. E. Humphreys, Linear algebraic groups, Graduate Texts in Mathematics, Vol. 21, SpringerVerlag, New York, Heidelberg, Berlin, 1987.

[JY] J. Jost and S.-T. Yau, A nonlinear elliptic system for maps from Hermitian to Riemannian manifolds and rigidity theorems in Hermitian geometry, Acta Math. 170 (1993), 221-254.

[LYZ] J. Li, S.-T. Yau and F. Zheng, On projectively flat Hermitian manifolds, Comm. Anal. Geom. 2 (1994), 103-109.

[Lo] J. Loftin, Affine Hermitian-Einstein metrics, Asian Jour. Math. 13 (2009), 101-130.

[Lu] M. Lübke, Chernklassen von Hermite-Einstein-Vektorbündeln, Math. Ann. 260 (1982), 133-141. 
[Ra] A. Ramanathan, Stable principal bundles on a compact Riemann surface, Math. Ann. 213 (1975), 129-152.

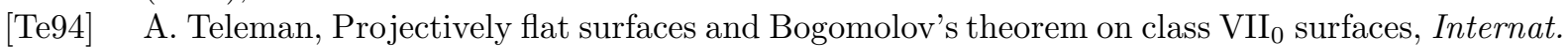
Jour. Math. 5 (1994), 253-264.

[Te05] A. Teleman, Donaldson theory on non-Kählerian surfaces and class VII surfaces with $b_{2}=1$, Invent. Math. 162 (2005), 493-521.

School of Mathematics, Tata Institute of Fundamental Research, Homi Bhabha Road, BOMBAY 400005, INDIA

E-mail address: indranil@math.tifr.res.in

Department of Mathematics and Computer Science, Rutgers University at Newark, NEwARK, NJ 07102, USA

E-mail address: loftin@rutgers.edu 